Article

\title{
Geometric Dynamics on Riemannian Manifolds
}

\author{
Constantin Udriste * (1) and Ionel Tevy (1) \\ Department of Mathematics-Informatics, Faculty of Applied Sciences, University Politehnica of Bucharest, \\ Splaiul Independentei 313, 060042 Bucharest, Romania; vascatevy@yahoo.fr or tevy@mathem.pub.ro \\ * Correspondence: anet.udri@yahoo.com or udriste@mathem.pub.ro
}

Received: 22 October 2019; Accepted: 23 December 2019; Published: 3 January 2020

\begin{abstract}
The purpose of this paper is threefold: (i) to highlight the second order ordinary differential equations (ODEs) as generated by flows and Riemannian metrics (decomposable single-time dynamics); (ii) to analyze the second order partial differential equations (PDEs) as generated by multi-time flows and pairs of Riemannian metrics (decomposable multi-time dynamics); (iii) to emphasise second order PDEs as generated by $m$-distributions and pairs of Riemannian metrics (decomposable multi-time dynamics). We detail five significant decomposed dynamics: (i) the motion of the four outer planets relative to the sun fixed by a Hamiltonian, (ii) the motion in a closed Newmann economical system fixed by a Hamiltonian, (iii) electromagnetic geometric dynamics, (iv) Bessel motion generated by a flow together with an Euclidean metric (created motion), (v) sinh-Gordon bi-time motion generated by a bi-flow and two Euclidean metrics (created motion). Our analysis is based on some least squares Lagrangians and shows that there are dynamics that can be split into flows and motions transversal to the flows.
\end{abstract}

Keywords: dynamical systems; generated ODEs and PDEs; single-time geometric dynamics; multi-time geometric dynamics; decomposable dynamics

MSC: 34A26; 35F55; 35G55

This is a synthesis paper that accumulates results obtained by our research group over time [1-8].

First, let us look for generating decomposable motions using flows (first order ODE system) and Riemannian metrics. In the multi-time case, things are a little more complicated since a set of $m \geq 2$ (eventually linearly independent) vector fields of class $C^{\infty}$ generates an $m$-dimensional distribution (first order ODE system or first order PDE system), and for the PDE system, we need pairs of Riemannian metrics.

\section{Statement of Geometric Dynamics Problems}

The subject of dynamical systems concerns the evolution of systems in single-time or multi-time (multivariate). In continuous single-time, the systems may be modeled by ODEs, PDEs, or other type of equations. In the case of ODEs the phase space is finite-dimensional and for PDEs the phase space is infinite-dimensional. In continuous multi-time, the systems may be modeled by PDEs or other type of equations.

The evolution parameter need not be the physical time or the time vector; for example, a time-stationary solution of a PDE is parameterized by spatial variables.

The fundamental problem discussed in this paper is threefold: (i) let us highlight again the technique of transforming a flow into dynamics by the geometry of the space in which the flow takes place, (ii) emphasize the decomposability of some important motions (dynamics) into flow trajectories and transversal to flow trajectories (depending on initial conditions); (iii) extend the previous ideas to harmonic maps between a source manifold and a target manifold. 
An 1-flow is generated by one $C^{\infty}$ vector field. A distribution of dimension $m$ is generated by $m$ vector fields of class $C^{\infty}$. If the distribution is completely integrable, then it is called $m$-flow. The geometry on a manifold is summarized by a Riemannian metric whose components play the role of gravitational potentials. Here, the focus is not on finding precise solutions to a given 1-flow or $m$-flow or $m$-distribution (which is often hopeless), but rather to answer questions like "can a flow or an $m$-flow or an $m$-distribution be changed into a dynamics and what is the main ingredient that accomplishes this?", or "can a dynamics be decomposable into flow trajectories and transversal to flow trajectories and what would be the structure of decomposability?".

By single-time dynamics we understand an ODE similar to the second law of Newton. A multi-time (multivariate) dynamics is described by a second order elliptic PDE.

Now we return to our previous theories, finding that it is important to emphasize the existence of decomposable movements and the necessary and sufficient conditions in which the decomposition takes place. The theory is general because in any sufficiently large dimension any ODE is transformed into an 1-flow or any PDE is transformed into an $m$-flow. The geometry of space transforms the 1-flow into a geodesic motion in a gyroscopic field of forces. The geometry of two spaces (source, target) transforms the $m$-flow (or integral manifolds of an $m$-distribution) into harmonic maps deformed by gyroscopic field of forces.

The first contribution of this paper is to propose a geometrical condition for expressing the impact between: (i) an 1-flow and the geometry of underlying manifold; (ii) an $m$-flow and the geometries of two (source and target) underlying manifolds; (iii) an $m$-distribution and the geometries of two (source and target) underlying manifolds. The second contribution of this paper is to use these well-formedness and strictness conditions to prove the decomposability of a motion. Our third contribution is to show the generality of our theory for writing ODEs and PDEs models whose set of solutions are decomposable. Topics include meaning of ODEs and PDEs on manifolds, both from geometric and systems theory view point. Also, least squares Lagrangians, and decomposable dynamics are included.

Although our Geometric Dynamics theory has deep connections with classical areas of mathematics such as the calculus of variations and the theory of ODEs or PDEs, it did not become a field in its own right until the late 2000s and early 2019s. At that time, some problems arising in engineering and economics were recognized as variants of problems in ODEs or PDEs and in the calculus of variations, although they were not covered by existing theories. Summing, our Geometric Dynamics is a branch of mathematics applying geometric methods to Dynamical Systems and the name was created for a talk at Second Conference of Balkan Society of Geometers, Aristotle University of Thessaloniki, 23-26 June 1998 and after for the book [2] and for the paper [3] (it was confirmed by J. Marsden who named it "geodesic motion in a gyroscopic field of forces", in the review "001k: 70016/MR70G45 (34A26 37C10 37J05 37K05 74E20)" made to our book [2]).

The name of Geometric Dynamics has been used by other authors for topics different than ours (see for example Proceedings of the International Symposium held at Instituto de Matematica Pura e Aplicada, Rio de Janeiro, Brasil, 1981 [9]; Workshop “Geometric Dynamics Days”, Ruhr-Universitat Bochum, Westfalische Wilhelms-Universitat Munster and the Technische Universitat Dortmund, 2013; and the paper [10], which investigates a family of dynamical systems arising from an evolutionary re-interpretation of certain optimal control and optimization problems).

Example 1. Equations of mechanics may appear different in form from $\dot{x}(t)=X(x(t))$, as they often involve higher time derivatives, but an equation that is second or higher order in time can always be rewritten as a set of first order equations.

The ODEs of the form $F(x(t), \dot{x}(t), \ddot{x}(t), \dddot{x}(t))=0$ which contain third order derivatives in them, are sometimes called jerk equations. It has been shown that a jerk equation is in a mathematically well defined sense 
the minimal setting for solutions showing chaotic behaviour. A jerk equation is equivalent to a system of three first-order ordinary non-linear differential equations

$$
\dot{x}(t)=y(t), \dot{y}(t)=z(t), \dot{z}(t)=\phi(x(t), y(t), z(t)) .
$$

This motivates a least squares Lagrangian of interest in jerk systems, namely

$$
2 L_{1}=(\dot{x}(t)-y(t))^{2}+(\dot{y}(t)-z(t))^{2}+(\dot{z}(t)-\phi(x(t), y(t), z(t)))^{2}
$$

on the jet space of coordinates $(t, x, y, z, \dot{x}, \dot{y}, \dot{z})$, and its associated geometric dynamics (Euler-Lagrange equations)

$$
\left.(\dot{z}-\phi) \phi_{x}+\ddot{x}-\dot{y}=0,(\dot{z}-\phi) \phi_{y}+\ddot{y}-\dot{z}=0,(\dot{z}-\phi) \phi_{z}+D_{t}(\dot{z}-\phi)\right)=0 .
$$

More generally, being given $n$ Lagrangians $L^{i}(t, x(t), \dot{x}(t)), i=\overline{1, n}, x(t)=\left(x^{1}(t), \ldots, x^{n}(t)\right), t \in I \subset$ $\mathbb{R}$, the associated least squares Lagrangian with respect to the Riemannian metric $g_{i j}(x)$ is

$$
\mathcal{L}=\frac{1}{2} g_{i j}(x(t)) L^{i}(t, x(t), \dot{x}(t)) L^{j}(t, x(t), \dot{x}(t)) .
$$

The extremals are solutions of the Euler-Lagrange ODE system

$$
\frac{1}{2} \frac{\partial g_{i j}}{\partial x^{k}} L^{i} L^{j}+g_{i j} L^{\partial} \frac{\partial L^{j}}{\partial x^{k}}-D_{t}\left(g_{i j} L^{i} \frac{\partial L^{j}}{\partial \dot{x}^{k}}\right)=0 .
$$

If the Lagrangian $L^{i}$ is associated to $O D E L^{i}(t, x(t), \dot{x}(t))=0$, then the extremals contain the solutions of that equation and the dynamics is decomposable.

Example 2. Let $u(x, t)$ be the density of the diffusing material at location $x \in \mathbb{R}^{n}$ and time $t \in \mathbb{R}$. Let $g^{i j}(u(x, t), x), i, j=\overline{1, n}$, be the collective diffusion coefficient (matrix) for density $u$ at location $x$. The diffusion $P D E$ is

$$
\frac{\partial u}{\partial t}(x, t)=\frac{\partial}{\partial x^{i}}\left(g^{i j}(u(x, t), x) \frac{\partial u}{\partial x^{j}}(x, t)\right) .
$$

If the diffusion coefficient depends on the density, then the diffusion equation is nonlinear, otherwise it is linear. More generally, when $g^{i j}(u(x, t), x)$ is a symmetric positive definite matrix (Riemannian metric), the equation describes anisotropic diffusion.

The diffusion PDE is equivalent to the first-order non-linear PDEs

$$
\frac{\partial u}{\partial x^{j}}=v_{j}, \frac{\partial u}{\partial t}=\frac{\partial}{\partial x^{i}}\left(g^{i j} v_{j}\right),
$$

where the parameter of evolution $(x, t)$ is $(n+1)$-dimensional. A Riemannian metric $h^{i j}(u(x, t), x)$ produces a least squares Lagrangian

$$
2 L_{2}=h^{i j}\left(\frac{\partial u}{\partial x^{i}}-v_{i}\right)\left(\frac{\partial u}{\partial x^{j}}-v_{j}\right)+\left(\frac{\partial u}{\partial t}-\frac{\partial}{\partial x^{i}}\left(g^{i j} v_{j}\right)\right)^{2},
$$

on the jet space of coordinates $\left(x, t, u, v, u_{x}, u_{t}, v_{x}, v_{t}\right)$. It appears the associated geometric dynamics (Euler-Lagrange equations)

$$
\begin{gathered}
\frac{1}{2} \frac{\partial h^{i j}}{\partial u}\left(\frac{\partial u}{\partial x^{i}}-v_{i}\right)\left(\frac{\partial u}{\partial x^{j}}-v_{j}\right)+\left(\frac{\partial u}{\partial t}-\frac{\partial}{\partial x^{i}}\left(g^{i j} v_{j}\right)\right)\left(-\frac{\partial}{\partial x^{i}}\left(\frac{\partial g^{i j}}{\partial u} v_{j}\right)\right) \\
-D_{x^{i}}\left(h^{i j}\left(\frac{\partial u}{\partial x^{j}}-v_{j}\right)\right)-D_{t}\left(\frac{\partial u}{\partial t}-\frac{\partial}{\partial x^{i}}\left(g^{i j} v_{j}\right)\right)=0,
\end{gathered}
$$




$$
g^{l m} D_{x^{m}}\left(\frac{\partial u}{\partial t}-\frac{\partial}{\partial x^{i}}\left(g^{i j} v_{j}\right)\right)=0 .
$$

Example 3. Let $T$ be an orientable manifold with the coordinates $t=\left(t^{1}, \ldots, t^{m}\right)$ and $M$ be a manifold with the coordinates $x=\left(x^{1}, \ldots, x^{n}\right)$. Using $m$ vector fields $X_{\alpha}(t, x)$ of class $C^{\infty}$ on $T \times M$, we introduce the distribution described by Pfaff equations

$$
d x^{i}(t)-X_{\alpha}^{i}(t, x) d t^{\alpha}=0, i=\overline{1, n}, \alpha=\overline{1, m} .
$$

Using some metric tensors $h_{\alpha \beta}(t), g_{i j}(t)$, and the components $\frac{\partial x^{i}}{\partial t^{\alpha}}(t)-X_{\alpha}^{i}(t, x)$ of the pullbacks, we build the least squares Lagrangian

$$
L=\frac{1}{2} g_{i j} h^{\alpha \beta}\left(\frac{\partial x^{i}}{\partial t^{\alpha}}(t)-X_{\alpha}^{i}(t, x)\right)\left(\frac{\partial x^{j}}{\partial t^{\beta}}(t)-X_{\beta}^{j}(t, x)\right) \sqrt{\operatorname{det}\left(h_{\alpha \beta}\right)}>0
$$

(non-decomposable dynamics).

Suppose the integral manifolds of the distribution have the dimension $1 \leq p<m$. Then we can introduce another least squares Lagrangian constructed from ODEs/PDEs that describes the integral manifolds and the action is an integral with the volume element on the $p$ parameters which define the integral manifold (decomposable dynamics).

More generally, being given $n m$ Lagrangians $L_{\alpha}^{i}\left(t, x(t), x_{\gamma}(t)\right), i=\overline{1, n}, \alpha=\overline{1, m}, x(t)=$ $\left(x^{1}(t), \ldots, x^{n}(t)\right), t=\left(t^{1}, \ldots, t^{m}\right) \in I \subset T$, then the associated least squares Lagrangian density with respect to the Riemannian metrics $g_{i j}(x) h^{\alpha \beta}(t), h^{\alpha \beta}(t)$ is

$$
\mathcal{L}=\frac{1}{2} g_{i j}(x(t)) h^{\alpha \beta}(t) L_{\alpha}^{i}\left(t, x(t), x_{\gamma}(t)\right) L_{\beta}^{j}\left(t, x(t), x_{\gamma}(t)\right) .
$$

If $T \subset \mathbb{R}^{m}$, the extremals are solutions of the Euler-Lagrange PDE system

$$
\frac{1}{2} \frac{\partial g_{i j}}{\partial x^{k}} h^{\alpha \beta} L_{\alpha}^{i} L_{\beta}^{j}+g_{i j} h^{\alpha \beta} L^{i} \frac{\partial L_{\beta}^{j}}{\partial x^{k}}-D_{\gamma}\left(g_{i j} h^{\alpha \beta} L_{\alpha}^{i} \frac{\partial L^{j}}{\partial x_{\gamma}^{k}}\right)=0 .
$$

If the Lagrangian $L_{\alpha}^{i}$ is associated to the PDE $L_{\alpha}^{i}\left(t, x(t), x_{\gamma}(t)\right)=0$, then the extremals contain the solutions of that equation and the dynamics is decomposable.

The ingredients needed to solve these problems are the Riemannian metrics, techniques of least squares Lagrangians and the idea of dynamics transversal decomposition.

The topics of the papers that inspired us in developing the theory of decomposable dynamics can be classified as follows:

Applied ODEs and PDEs [11-14] are evolution equations modeling systems evolving with respect to a "time" parameter. When solving such evolution equations, the appropriate formulation of the problem is usually as an initial value, or Cauchy, problem. More specifically, certain initial data are given, representing the state of the system at some initial time. The goal, then, is to "predict the future", that is, to find the solution of the ODE or PDE, which represents the behaviour of the system at all moments.

Hamiltonian approach [15-17] of dynamics is sometimes more subtle than the Lagrangian approach because this point of view changes the dynamics into a Hamiltonian flow. Hamiltonian dynamics are based on ODEs or PDEs of the first order constructed from ODEs or PDEs of second order Euler-Lagrange equations. The transition from the Euler-Lagrange-type equations to Hamilton-type first order equations is based on the Legendre transformation.

Dynamics, winds and flows $[18,19]$ are often mysteriously coupled. The most interesting cases are those of decomposable dynamics or winds. 
Variational principles [20] are alternative methods for determining the state or dynamics of a physical system, by identifying it as a critical point (minimum, maximum or saddle point) of a functional.

Combining the previous ideas with the thought that certain flows accompanied by space geometry (Riemannian metric and Riemannian connection - derivation), generate what is now called the geometric dynamics. This geometric dynamics have been discovered by us first for magnetic flow and then for any other flow. It is in fact a geodesic motion in a gyroscopic field of forces. In time the ideas were extended by our research team to $m$-flows and to $m$-distributions [1-8].

The main aim of this paper is to give necessary and sufficient conditions for the decomposition of a general (single-time or multi-time) dynamics into a flow and a transversal movement.

\section{Single-Time Geometric Dynamics}

\subsection{First and Second Order ODEs on Manifolds}

Let $M$ be a differentiable manifold and $I \subset \mathbb{R}$ be a nontrivial interval.

A (time dependent) non-autonomous first order differential equation on a manifold $M$ [13] is given by assigning, on an open subset $V$ of $\mathbb{R} \times M$, a non-autonomous $C^{\infty}$ vector field $X: V \rightarrow \mathbb{R}^{n}$, which is tangent to $M$ for all $t \in \mathbb{R}$. That is, for any $t \in \mathbb{R}$, the map $X_{t}: V_{t} \rightarrow \mathbb{R}^{n}$, given by $X_{t}(x)=X(t, x)$, is a tangent vector field on the (possibly empty) open subset $V_{t}=\{x \in M \mid(t, x) \in V\}$ of $M$. In other words, $X(t, x) \in T_{x} M$ for each $(t, x) \in V$ and the first order differential equation associated to $X$ is defined by

$$
\dot{x}=X(t, x),(t, x) \in V .
$$

A solution of the differential Equation (1) is a $C^{1}$ map $x: I \rightarrow M$, such that, for all $t \in I,(t, x(t)) \in$ $V$ and $\dot{x}(t)=X(t, x(t))$, identically on $I$. The Cauchy problem is the following: find a solution of the ODE (1) which satisfies the initial condition $x\left(t_{0}\right)=x_{0}$. Answer: the solution of this Cauchy problem exists and it is unique.

Let $F: \mathbb{R} \times T M \rightarrow \mathbb{R}^{n}$ be a $C^{\infty}$ map. An equality of the type

$$
\ddot{x}=F(t, x, \dot{x}),(t, x, \dot{x}) \in \mathbb{R} \times T M
$$

is called a (time dependent) second order differential equation on $M$ [13], provided that the associated vector field

$$
G: \mathbb{R} \times T M \rightarrow \mathbb{R}^{n} \times \mathbb{R}^{n}, G(t, x, y)=(y, F(t, x, y))
$$

is tangent to $T M$, i.e., $(y, F(t, x, y)) \in T_{(x, y)} T M$ for all $(t, x, y) \in \mathbb{R} \times T M$. A solution of the differential Equation (2) is a $C^{2}$ curve $x: I \rightarrow \mathbb{R}^{n}$, in such a way that $x(t) \in M$ and $\ddot{x}(t)=F(t, x(t), \dot{x}(t))$, identically on $I$. The Cauchy problem: find a solution of the ODE (2) which satisfies the initial conditions $x\left(t_{0}\right)=x_{0}, \dot{x}\left(t_{0}\right)=v$. Answer: the solution of this Cauchy problem exists and it is unique.

If we use the components, the relations (1) and (2) are called respectively first order and second order ODE systems.

\subsection{Single-Time Geometric Dynamics}

We start with the triple $(M, g, X)$, where $M$ is a manifold of dimension $n, g(x)=\left(g_{i j}(x)\right), i, j=$ $1, \ldots, n$, is a Riemannian metric and $X(t, x)=\left(X^{i}(t, x)\right)$ a time dependent $C^{\infty}$ vector field, on the manifold $M$. Suppose the Levy-Civita connection $\nabla$ of $(M, g)$ has the components $G_{j k}^{i}, i, j, k=1, \ldots, n$.

Definition 1. We use the notations

$$
F_{j}=\left(F_{j}^{i}\right), F_{j}^{i}=\nabla_{j} X^{i}-g^{i h} g_{k j} \nabla_{h} X^{k}, f=\frac{1}{2} g(X, X) .
$$


A function $F: \mathbb{R} \times T M \rightarrow \mathbb{R}^{n}$ is said to be generated by the pair $(X, g)$ if it is of the form

$$
F=-G_{j k} \dot{x}^{j} \dot{x}^{k}+F_{j} \dot{x}^{j}+\nabla f+\frac{\partial}{\partial t} X .
$$

If $F$ is generated by $X$ and $g$, then the ODE (2) represents a single-time geometric dynamics or a geodesic motion in a gyroscopic field of forces [1-8]. By analogy with the reduction of the force system in mechanics, resultant and momentum, the decomposition of the set of solutions returns to the flow and the movement in the gyroscopic field of forces.

Theorem 1. If $F: \mathbb{R} \times T M \rightarrow \mathbb{R}^{n}$ is generated by the pair $(X, g)$, then the set of maximal solutions of ODE (2) is decomposable into a subset corresponding to the initial values

$$
x\left(t_{0}\right)=x_{0}, \dot{x}\left(t_{0}\right)=X\left(t_{0}, x\left(t_{0}\right)\right),
$$

solutions which are reducible to solutions of the ODE (1), and a subset of solutions corresponding to the initial values

$$
x\left(t_{0}\right)=x_{0}, \dot{x}\left(t_{0}\right)=W \neq \lambda X\left(t_{0}, x\left(t_{0}\right)\right), \lambda>0,
$$

transversal to the solutions of the ODE (1). The converse is also true.

Proof. Based on existence and uniquennes theorem, each solution $x=x(t)$ of any second order prolongation of first order ODE system has the property: $\dot{x}\left(t_{0}\right)=X\left(t_{0}, x\left(t_{0}\right)\right)$ implies $\dot{x}(t)=$ $X(t, x(t)), \forall t \in I$.

A flow $X$ and a Riemannian metric $g$ determines a least squares Lagrangian

$$
L(t, x, \dot{x})=\frac{1}{2} g(\dot{x}-X(t, x), \dot{x}-X(t, x))
$$

The Euler-Lagrange ODEs represent a geometric prolongation of the flow.

The Euler-Lagrange ODEs constitute just a decomposable dynamics (geodesic motion in a gyroscopic fields of forces) $\equiv$ (set of flow trajectories) $\oplus$ (set of transversal trajectories imposed by the geometry of the space).

Theorem 2. Suppose that $X$ is an autonomous vector field. If the function $F: T M \rightarrow R^{n}$ is generated by $X$ and $g$, then the set of maximal solutions of ODE (2) splits into three categories:

(i) curves $x(t)$ characterized by $H(x(t))=$ const $=0$;

(ii) curves $x(t)$ characterized by $H(x(t))=$ const $>0$;

(iii) curves $x(t)$ characterized by $H(x(t))=$ const $<0$.

Proof. These statements are based on the Hamiltonian

$$
H(t, x, \dot{x})=\frac{1}{2} g(\dot{x}-X(t, x), \dot{x}+X(t, x))=\frac{1}{2}(g(\dot{x}, \dot{x})-g(X, X))=H(x, \dot{x}),
$$

and the associated Hamilton ODEs.

The curves $x(t)$ with $H(x(t))=$ const $=0$ are solutions of ODE (1). The solutions with $H(x(t))=$ const $\neq 0$, are transversal to solutions of ODE (1).

Remark 1. (i) Any normal ODE generates in the phase space a flow, which together with the phase space geometry give a geometric dynamics. This statement is true for any ODE, but then appears a flow with constraint. 
(ii) Let us consider the quadruple $(M, X, g, \Gamma)$, where $M$ is a manifold, $X$ is a flow on $M, g$ is a fundamental tensor field and $\Gamma$ is a symmetric connection (derivation). The triple $(X, g, \Gamma)$ generates an extended geometric dynamics on $M$ determined by ODEs

$$
\ddot{x}^{i}(t)=\left(\delta_{k}^{i} \delta_{j}^{l}-g_{k j} g^{l i}\right) X_{, l}^{k} \dot{x}^{j}(t)+\frac{\partial X^{i}}{\partial t}+g_{k j} g^{l i} X_{, l}^{k} X^{j}
$$

(iii) On the Riemannian manifold $((0, \infty), g(x)=1)$, let us take the flow $\dot{x}=1$. We attach the least squares Lagrangian $L_{1}=(\dot{x}-1)^{2}$, with Euler-Lagrange equation $\ddot{x}=0$. On any other Riemannian manifold $((0, \infty), g(x))$, we find the least squares Lagrangian $L_{2}=g(x)(\dot{x}-1)^{2}$, with Euler-Lagrange equation $\ddot{x}=\frac{g^{\prime}(x)}{2 g(x)}(1-\dot{x})(1+\dot{x})$. Here, $\Gamma(x)=\frac{g^{\prime}(x)}{2 g(x)}$ is a linear connection. We can extend the previous ODE to the ODE system

$$
\ddot{x}^{i}(t)=a_{0}^{i}(x(t))+a_{j}^{i}(x(t)) \dot{x}^{j}(t)+b_{j k}^{i}(x(t)) \dot{x}^{j}(t) \dot{x}^{k}(t), i, j, k=1, \ldots, n,
$$

with possible chaos in velocities.

\subsection{Fundamental Tensor Field}

Let $M$ be a differentiable manifold of dimension $n$ and $I \subset \mathbb{R}$ be a nontrivial interval. If the ODE system (2) is an Euler-Lagrange system on $M$ for a regular Lagrangian $L(t, x, \dot{x})$, then there exists a fundamental $d$-tensor field $g=\left(g_{i j}\right)$ on TM such that

$$
g_{i j}(t, x, \dot{x})=\frac{1}{2} \frac{\partial^{2} L}{\partial \dot{x}^{i} \partial \dot{x}^{j}}(t, x, \dot{x}), i, j=1, \ldots, n .
$$

Conversely, given $g_{i j}(t, x, \dot{x})$, to determine $L(t, x, \dot{x})$, we need complete integrability conditions. In these conditions, using two successive curvilinear integrals of the second type, we can write

$$
L(t, x, \dot{x})=\int_{\gamma_{\dot{x}_{0} \dot{x}}} \int_{\gamma_{\dot{x}_{0} \dot{x}}} g_{i j}(t, x, \dot{x}) d \dot{x}^{i} d \dot{x}^{j}+a_{i}(t, x) \dot{x}^{i}+b(t, x) .
$$

The pair $(M, g)$ is called a Lagrangian manifold.

\section{First Examples of Generated Geometric Dynamics}

Let us show that the movement of planets and motion in closed Newmann economical systems are generated by flows and Riemannian metrics.

Given a function $u(x), x \in M$ and a Riemannian metric $g$ on $M$, let us consider the Hamiltonian $H=\frac{1}{2} g(\dot{x}, \dot{x})-u(x)$, as in [5]. If $u(x)>0$, then the vector field $X(x)=\sqrt{2 u(x)} E(x)$ (Galilei formula), where $E$ is an arbitrary unit vector field with respect to the metric $g$, satisfies $g(X, X)=2 u(x)$. Consequently such a Hamiltonian, equal to the difference between the kinetic energy and a positive function, is coming from a vector field (flow) and a Riemannian metric, corresponding to a perfect square Lagrangian.

Theorem 3. If $u$ is positive, then the motion described by the Hamiltonian $H=\frac{1}{2} g(\dot{x}, \dot{x})-u(x)$ is generated by a flow and a Riemannian metric.

\subsection{Motion of the Four Outer Planets}

Let $m_{1}, m_{2}, m_{3}, m_{4}$ be the masses of the four outer planets (Jupiter, Saturn, Uranus, Neptune), relative to the sun mass $m_{0}=1$. They produce an Euclidean metric $g=\operatorname{diag}\left(m_{0}, m_{1}, m_{2}, m_{3}, m_{4}\right)$.

Corollary 1. The motion of the four outer planets relative to the sun is generated by a flow $E(q)$ and the Euclidean metric g. 
Proof. The motion of the four outer planets relative to the sun is described by the Hamiltonian

$$
H(p, q)=\frac{1}{2} \sum_{i=0}^{4} \frac{1}{m_{i}}\left\|p_{i}\right\|^{2}-G \sum_{i=1}^{4} \sum_{j=0}^{i-1} \frac{m_{i} m_{j}}{\left\|q_{i}-q_{j}\right\|^{\prime}}
$$

where $p=\left(p_{0}, p_{1}, p_{2}, p_{3}, p_{4}\right)$ and $q=\left(q^{0}, q^{1}, q^{2}, q^{3}, q^{4}\right)$ are velocity and position (supervectors) with $p_{i}, q^{i} \in \mathbb{R}^{3}, G$ is the gravitational constant, and $m_{1}, m_{2}, m_{3}, m_{4}$ are masses relative to the sun mass $m_{0}=1$. We apply the previous statement with a generic versor

$$
\begin{gathered}
E(q)=\left(\frac{1}{\sqrt{m_{0}}} \cos \varphi_{0}, \frac{1}{\sqrt{m_{1}}} \sin \varphi_{0} \cos \varphi_{1}, \frac{1}{\sqrt{m_{1}}} \sin \varphi_{0} \sin \varphi_{1} \cos \varphi_{2},\right. \\
\left.\frac{1}{\sqrt{m_{3}}} \sin \varphi_{0} \sin \varphi_{1} \sin \varphi_{2} \cos \varphi_{3}, \frac{1}{\sqrt{m_{4}}} \sin \varphi_{0} \sin \varphi_{1} \sin \varphi_{2} \sin \varphi_{3}\right),
\end{gathered}
$$

where $\varphi_{i}=\varphi_{i}(q)$. One associated flow requires the fixing of the versor field $E(q)$.

Since these planets are rather large, their orbits can affect one another (and possibly even the Sun).

\subsection{Motion in Closed Newmann Economical Systems}

A closed economical system is one that has no trade activity with outside economies. The closed economical system is self-sufficient, that means no imports come into the system and no exports leave the system.

Corollary 2. The motion in closed Newmann economical systems is generated by a flow and a Riemannian metric $g_{i j}$.

Proof. We use a Hamiltonian [21,22] which relates $n$ capital goods $K_{1}, \ldots, K_{n}$ and the net capital formations $\dot{K}_{1}, \ldots, \dot{K}_{n}$, namely

$$
H(K, \dot{K})=g^{i j}(K) \dot{K}_{i} \dot{K}_{j}-K_{1}^{\alpha_{1}} \cdots K_{n}^{\alpha_{n}}, \alpha_{1}+\cdots+\alpha_{n}=1 .
$$

We apply the previous statement with a generic versor field.

\section{Comparison between Lorentz's Law and Geometric Dynamics}

\section{Lorentz law}

Let $x=\left(x^{1}, x^{2}, x^{3}\right) \in \mathbb{R}^{3}$ and $t \in \mathbb{R}$. For the potential vector magnetic field $A(x, t)$, a particle moving with velocity $v=\dot{x}$ and particle charge $e$ has the potential momentum $e A(x, t)$, so its potential energy is $e A(x, t) \cdot \dot{x}$. For a $\phi(x, t)$ field, the particle's potential energy is $e \phi(x, t)$. Using the total potential energy $V=e \phi-e A \cdot \dot{x}$ and the kinetic energy $T=\frac{m}{2} \dot{x} \cdot \dot{x}$, we built the Lorentz Lagrangian

$$
L_{1}=T-V=\frac{m}{2} \dot{x} \cdot \dot{x}+e A \cdot \dot{x}-e \phi .
$$

It is well-known that the movement of a charged particle into an electromagnetic field is described by the Euler-Lagrange ODE system (universal Lorentz law)

$$
m \ddot{x}=e(E+\dot{x} \times \operatorname{rot} A) .
$$

The Lorentz Hamiltonian is

$$
H_{L_{1}}=\frac{m}{2} \dot{x} \cdot \dot{x}+e \phi .
$$

\section{Geometric dynamics}


For the preservation of traditional formulas, we will refer to the magnetic flow generated by the vector potential " $-A$ ", using homogeneous dimensional relationships. The magnetic trajectories are the solutions of the ODE system $m \dot{x}=-e A$. This system together with the Euclidean metric produce the least squares Lagrangian

$$
L_{2}=\frac{1}{2}(m \dot{x}+e A) \cdot(m \dot{x}+e A) .
$$

The Euler-Lagrange ODEs of $L_{2}$ are

$$
m \ddot{x}=e \dot{x} \times \operatorname{rot} A+\frac{e^{2}}{m} \nabla f_{A}-e \partial_{t} A,
$$

where

$$
f_{A}=\frac{1}{2}\|A\|^{2}
$$

is the energy density associated to the vector field $A$. In this way we obtain a single-time geometric dynamics, which is in fact a geodesic motion in a gyroscopic field of forces. The associated Hamiltonian is

$$
H_{2}=\frac{m^{2}}{2} \dot{x} \cdot \dot{x}-e^{2} f_{A} .
$$

Remark 2. (i) Generally, the single-time geometric dynamics produced by the potential vector field " $-A$ " is different from the classic universal Lorentz law because

$$
L_{2}-m L_{1}=\frac{1}{2} e^{2} A \cdot A+m e \phi
$$

and the force field $\frac{e}{m} \nabla f_{A}-\partial_{t} A$ is not the electric field $E=-\nabla \phi-\partial_{t} A$. In other words the Lagrangians $L_{1}$ and $L_{2}$ are not in the same equivalence class of Lagrangians.

(ii) The magnetic force $F=e \dot{x} \times \operatorname{rot} A$ do no work on the moving charge, being a gyroscopic force (the mechanical work produced by $F$ is zero). Any gyroscopic force has the same property.

\section{Geometric Dynamics Induced by Bessel Kinematics}

Let ODE be an arbitrary differential equation and $g$ be a Riemannian metric. The pair (ODE, $g$ ) generates a geometric dynamics.

Of course, we are interested in meaningful pairs. For example, let us start by recalling the Bessel ODE,

$$
\ddot{x}(t)+\frac{1}{t} \dot{x}(t)+\left(1-\frac{\alpha^{2}}{t^{2}}\right) x(t)=0, t \in(0, \infty) .
$$

It is well known that Bessel functions are very important in many problems of wave propagation and static potentials. The associated Bessel flow (kinematics) is

$$
\dot{x}=y, \quad \dot{y}=-\frac{1}{t} y-\left(1-\frac{\alpha^{2}}{t^{2}}\right) x,
$$

on the Riemannian manifold $\left(\mathbb{R}^{2}, \delta_{i j}\right)$. Consequently the associated least squares Lagrangian is

$$
L=\frac{1}{2}\left[(\dot{x}-y)^{2}+\left(\dot{y}+\frac{1}{t} y+\left(1-\frac{\alpha^{2}}{t^{2}}\right) x\right)^{2}\right] .
$$

By a direct computation, we get the corresponding Euler-Lagrange equations

$$
\ddot{x}=\left(2-\frac{\alpha^{2}}{t^{2}}\right) \dot{y}+\frac{1}{t}\left(1-\frac{\alpha^{2}}{t^{2}}\right) y+\left(1-\frac{\alpha^{2}}{t^{2}}\right)^{2} x
$$




$$
\ddot{y}=\left(1+\frac{2}{t^{2}}\right) y-\left(2-\frac{\alpha^{2}}{t^{2}}\right) \dot{x}+\left(\frac{1}{t}-\frac{3 \alpha^{2}}{t^{3}}\right) x .
$$

These ODEs are the same as the Bessel geometric dynamics ODEs

$$
\frac{d^{2} x^{i}}{d t^{2}}=\frac{\partial X^{i}}{\partial t}+\frac{\partial f}{\partial x^{i}}+\sum_{j=1}^{2}\left(\frac{\partial X^{i}}{\partial x^{j}}-\frac{\partial X^{j}}{\partial x^{i}}\right) \frac{d x^{j}}{d t}, \quad i=1,2,
$$

where $x^{1}:=x, x^{2}:=y$ and $f(t, x, y):=\frac{1}{2}\|X\|^{2}$ is the energy of the vector field

$$
X=\left(X^{1}, X^{2}\right), X^{1}(t, x, y):=y, \quad X^{2}(t, x, y):=-\frac{1}{t} y-\left(1-\frac{\alpha^{2}}{t^{2}}\right) x
$$

A direct calculation in (4) gives us the Euler-Lagrange ODEs. Therefore, we have

Theorem 4. (see [5,7]) The second order ODEs system (4) is an Euler-Lagrange prolongation of the first order ODEs system (3) if and only if the Lagrangian L is of least squares type, modulo a total derivative term.

\section{Multi-Time Geometric Dynamics}

\subsection{First and Second Order PDEs on Manifolds}

We start with a triple $\left((T, h),(M, g), X_{\alpha}\right)$, where: $(i)(T, h)$ is an oriented Riemannian manifold (source space) of dimension $m$, with local coordinates $t=\left(t^{\alpha}\right), \alpha=1, \ldots, m$, metric tensor $h_{\alpha \beta}$ and Christoffel symbols $H_{\beta \gamma}^{\alpha}$; (ii) $(M, g)$ is a Riemannian manifold (target space) of dimension $n$, with local coordinates $x=\left(x^{i}\right), i=1, \ldots, n$, metric tensor $g_{i j}$ and Christoffel symbols $G_{j k}^{i} ;($ iii $) X_{\alpha}(t, x)=$ $\left(X_{\alpha}^{i}(t, x)\right), \alpha=1, \ldots, m ; i=1, \ldots, n$ are $C^{\infty}$ vector fields on $M$, dependent on $(t, x)$, which define the first order PDE system

$$
\frac{\partial x}{\partial t^{\alpha}}(t)=X_{\alpha}(t, x(t))
$$

Theorem 5. (existence and uniqueness) [20] The Cauchy problem consisting in the PDE system (5) and the initial condition $x\left(t_{0}\right)=x_{0}$ has a unique solution if and only if the system is completely integrable.

An equality of the type

$$
h^{\alpha \beta} \frac{\partial^{2} x}{\partial t^{\alpha} \partial t^{\beta}}(t)=F\left(t, x(t), x_{\gamma}(t)\right),\left(t, x, x_{\gamma}\right) \in J^{1}(T, M)
$$

is called a (time dependent) second order elliptical PDE (system) on $M$.

Let $\Gamma: G(t)=0$ be a hypersurface in $T$, containing the point $t_{0}$ and $\Lambda(t)$ be a unit vector field along $\Gamma$, transversal (non-tangent) to $\Gamma$. Denote $\varphi_{0}(t)$ and $\varphi_{1}(t)$ as vector functions with $n$ components on $\Gamma$, the first being of class $C^{1}$ and the second of class $C^{0}$. The Cauchy problem attached to PDE (6) is (see [14], p. 208): find, in an unilateral or bilateral neighborhood of $\Gamma$, the solution of the PDE (6) satisfying the Cauchy conditions

$$
\left.x(t)\right|_{\Gamma}=\varphi_{0}(t),\left.D_{\Lambda} x(t)\right|_{\Gamma}=\varphi_{1}(t)
$$

Answer: the solution of this Cauchy problem exists and it is unique.

Knowing the Cauchy conditions, one can find the values of all first order partial derivatives of the function $x(t)$ on the Cauchy surface $\Gamma$ : firstly,

$$
\left.\frac{\partial x}{\partial t^{\alpha}}\right|_{\Gamma}=\frac{\partial \varphi_{0}}{\partial t^{\alpha}}(t), \alpha=1, \ldots, m-1
$$


and then the equalities

$$
\varphi_{1}(t)=\left.D_{\Lambda} x(t)\right|_{\Gamma}=\frac{\partial x}{\partial t^{\alpha}}(t) \Lambda^{\alpha}(t)
$$

together with $\Lambda^{m} \neq 0$, give

$$
\left.\frac{\partial x}{\partial t^{m}}(t)\right|_{\Gamma}=\frac{1}{\Lambda^{m}(t)}\left[\varphi_{1}(t)-\sum_{\alpha=1}^{m-1} \frac{\partial \varphi_{0}}{\partial t^{\alpha}}(t) \Lambda^{\alpha}(t)\right] .
$$

Lemma 1. The initial conditions (7) are equivalent either to the initial conditions

$$
\text { (i) }\left.x(t)\right|_{\Gamma}=\varphi_{0}(t),\left.\frac{\partial x}{\partial t^{m}}(t)\right|_{\Gamma}=W_{m}(t)
$$

or to

$$
\text { (ii) }\left.x(t)\right|_{\Gamma}=\varphi_{0}(t),\left.\frac{\partial x}{\partial t^{\alpha}}(t)\right|_{\Gamma}=W_{\alpha}(t), \alpha=1, \ldots, m,
$$

together with the complete integrability conditions and the compatibility condition to $\varphi_{0}$.

\subsection{Multi-Time Geometric Dynamics}

The multi-time geometric dynamics was introduced in our papers $[1,5,8]$ like Multi-time World Force Law involving field potentials (components of the $d$-tensor), gravitational potentials (components of the two Riemannian metrics), and the Yang-Mills potentials (components of the Riemannian connections and the nonlinear connection). This evolution can be called also harmonic maps deformation in a gyroscopic field of forces.

Definition 2. Using the vector fields $X_{\alpha}$, the metric tensors $h_{\alpha \beta}, g_{i j}$, and the Christoffel symbols $H_{\beta \gamma^{\prime}}^{\alpha} G_{j k^{\prime}}^{i}$ we define

$$
\begin{gathered}
F_{j \alpha}^{i}=\nabla_{j} X_{\alpha}^{i}-g^{i h} g_{k j} \nabla_{h} X_{\alpha}^{k}, f=\frac{1}{2} h^{\alpha \beta} g_{i j} X_{\alpha}^{i} X_{\beta}^{j} \\
\nabla_{j} X_{\alpha}^{i}=\frac{\partial X_{\alpha}^{i}}{\partial x^{j}}+G_{j k}^{i} X_{\alpha}^{k}, D_{\beta} X_{\alpha}^{i}=\frac{\partial X_{\alpha}^{i}}{\partial t^{\beta}}-H_{\alpha \beta}^{\gamma} X_{\gamma}^{i} .
\end{gathered}
$$

The function $F: J^{1}(T, M) \rightarrow \mathbb{R}^{n}$ is said to be generated by the triplet $\left(X_{\alpha}, h, g\right)$ if it is of the form

$$
F=h^{\alpha \beta}\left(-G_{j k} x_{\alpha}^{j} x_{\beta}^{k}+H_{\alpha \beta}^{\gamma} x_{\gamma}+F_{j \alpha} x_{\beta}^{j}+g_{k j}\left(\nabla X_{\alpha}^{k}\right) X_{\beta}^{j}+D_{\beta} X_{\alpha}\right) .
$$

If $F$ is generated by $X_{\alpha}, h$ and $g$, then the PDE (4) represents a multi-time geometric dynamics.

Suppose that the PDE system (3) is completely integrable ( $m$-flow).

Theorem 6. If $F: J^{1}(T, M) \rightarrow \mathbb{R}^{n}$ is generated by the triplet $\left(X_{\alpha}, h, g\right)$, then the set of maximal solutions of $P D E(6)$ is decomposable into a subset corresponding to the initial values

$$
\left.x(t)\right|_{\Gamma}=\varphi_{0}(t),\left.\frac{\partial x}{\partial t^{\alpha}}(t)\right|_{\Gamma}=X_{\alpha}(t, x(t)),
$$

solutions which are reducible to solutions of PDE (5), and a subset of solutions corresponding to the initial values

$$
\left.x(t)\right|_{\Gamma}=\varphi_{0}(t),\left.\frac{\partial x}{\partial t^{\alpha}}(t)\right|_{\Gamma}=W_{\alpha}(t) \notin K^{+}\left\{X_{\alpha}(t, x(t))\right\},
$$

transversal to the solutions of PDE (5). The converse is also true.

Proof. Each solution $x=x(t)$ of any second order prolongation of the first order PDE system has the property: $x_{\alpha}\left(t_{0}\right)=X_{\alpha}\left(t_{0}, x\left(t_{0}\right)\right)$ implies $x_{\alpha}(t)=X_{\alpha}(t, x(t)), \forall t \in T$. 
Any $m$-flow $X_{\alpha}$ and two Riemannian metrics $h$ and $g$ determine a least squares Lagrangian density

$$
L\left(t, x, x_{\gamma}\right)=\frac{1}{2} h^{\alpha \beta} g_{i j}\left(x_{\alpha}^{i}-X_{\alpha}^{i}(t, x)\right)\left(x_{\beta}^{j}-X_{\beta}^{j}(t, x)\right) \text {. }
$$

The Euler-Lagrange PDEs represent a prolongation of the $m$-flow and just a decomposable dynamics.

Remark 3. (i) Any normal PDE generates in the phase space a multidimensional flow, which together with the phase space geometry gives a geometric dynamics. This statement is true for any PDE, but then appears a multidimensional flow with constraints.

(ii) Let us consider the triple $(T, h, H)$, where $T$ is a manifold, $h$ is a fundamental tensor field and $H$ is a symmetric connection (derivation). We add the quadruple $\left(M, X_{\alpha}, g, G\right)$, where $M$ is a manifold, $X_{\alpha}$ is an $m$-flow on $M, g$ is a fundamental tensor field and $G$ is a symmetric connection (derivation). The quintuple $\left(X_{\alpha} ; h, H ; g, G\right)$ generates an extended geometric dynamics on $T \times M$.

\subsection{Fundamental Tensor Field}

Let $M$ be a differentiable manifold of dimension $n$ and $I \subset \mathbb{R}^{m}$ be a nontrivial interval. If the ODE system (4) is an Euler-Lagrange system on $M$ for a regular Lagrangian $L\left(t, x, x_{\gamma}\right)$, then there exists a fundamental tensor field $G=\left(G_{i j}^{\alpha \beta}\right)$ such that

$$
G_{i j}^{\alpha \beta}\left(t, x, x_{\gamma}\right)=\frac{1}{2} \frac{\partial^{2} L}{\partial x_{\alpha}^{i} \partial x_{\beta}^{j}}\left(t, x, x_{\gamma}\right), i, j=1, \ldots, n ; \alpha, \beta=1, \ldots, m .
$$

Conversely, given $G_{i j}^{\alpha \beta}\left(t, x, x_{\gamma}\right)$, to determine $L\left(t, x, x_{\gamma}\right)$, we need complete integrability conditions. In these conditions, using two successive curvilinear integrals of the second type, we can write

$$
L\left(t, x, x_{\gamma}\right)=\int_{\left(x_{\alpha}^{i}\right)_{0}}^{\left(x_{\alpha}^{i}\right)} \int_{\left(x_{\beta}^{j}\right)_{0}}^{\left(x_{\beta}^{j}\right)} G_{i j}^{\alpha \beta}\left(t, x, x_{\gamma}\right) d x_{\alpha}^{i} d x_{\beta}^{j}+a_{i}^{\alpha}(t, x) x_{\alpha}^{i}+b(t, x) .
$$

The fundamental tensor field is said to be Kronecker decomposable if $G=h \otimes g$. The pair $(M, G)$ is called a Lagrangian manifold.

\section{Geometric Dynamics Induced by sinh-Gordon Kinematics}

This Section was elaborated in our research group.

Any triple (PDE, $h, g)$ generates a geometric dynamics, but we are interested in meaningful triples. For example, we use the sinh-Gordon equation $\frac{\partial^{2} u}{\partial x \partial t}(x, t)=\sinh u(x, t)$. The sinh-Gordon equation is a nonlinear partial differential equation that has applications in physics and hydrodynamics. It is known for its soliton solutions and arises as a special case of the Toda lattice equation.

Case 1 The equivalent sinh-Gordon diagonal flow (kinematics) is given by

$$
u_{x}=v, \quad v_{t}=\sinh u
$$

We attach a least squares Lagrangian

$$
L_{1}=\frac{1}{2}\left[\left(u_{x}-v\right)^{2}+\left(v_{t}-\sinh u\right)^{2}\right]
$$

on the Riemannian manifolds $\left(\mathbb{R}^{2}, \delta^{\alpha \beta}\right)$ and $\left(\mathbb{R}^{2}, \delta_{i j}\right)$. Its Euler-Lagrange PDEs are

$$
u_{x x}=v_{x}-\left(v_{t}-\sinh u\right) \cosh u, v_{t t}=u_{t} \cosh u-u_{x}+v .
$$


Case 2 A complete sinh-Gordon flow (kinematics) is given by

$$
u_{x}=v, v_{t}=\sinh u, u_{t}=f, v_{x}=g
$$

This PDE system is completely integrable if and only if $f_{x}=\sinh u, g_{t}=u_{x} \cosh u$.

We use the least squares Lagrangian

$$
L_{2}=\frac{1}{2}\left[\left(u_{x}-v\right)^{2}+\left(v_{t}-\sinh u\right)^{2}+\left(u_{t}-f\right)^{2}+\left(v_{x}-g\right)^{2}\right]
$$

on the Riemannian manifolds $\left(\mathbb{R}^{2}, \delta^{\alpha \beta}\right)$ and $\left(\mathbb{R}^{2}, \delta_{i j}\right)$. The Euler-Lagrange equations associated to this Lagrangian are

$$
\begin{gathered}
u_{t t}+u_{x x}=\sinh u \cosh u-v_{t} \cosh u+f_{t}+v_{x}, \\
v_{t t}+v_{x x}=v+u_{t} \cosh u+g_{x}-u_{x} .
\end{gathered}
$$

If the PDE system (10) is completely integrable, then the set of solutions of the Euler-Lagrange PDEs includes the set of solutions of PDE (10); otherwise, it does not. In the case when the PDE system (10) is not completely integrable, the solutions of the Euler-Lagrange PDE are solutions of the least square approximation for PDE (10).

On the other hand, the sinh-Gordon geometric dynamics is described by (see definition)

$$
h^{\alpha \beta}\left(\frac{\partial^{2} x}{\partial t^{\alpha} \partial t^{\beta}}+G_{j k} x_{\alpha}^{j} x_{\beta}^{k}-H_{\alpha \beta}^{\gamma} x_{\gamma}-F_{j \alpha} x_{\beta}^{j}-g_{k j}\left(\nabla X_{\alpha}^{k}\right) X_{\beta}^{j}-D_{\beta} X_{\alpha}\right)=0 .
$$

Therefore, we have

Theorem 7 (see [5,7]). This last second order PDEs system is an Euler-Lagrange prolongation of the first order PDEs system (10) if and only if the Lagrangian L is of least squares type, modulo a divergence type term.

\section{How Are Disasters Favored?}

Besides developing theories on ODE and PDE systems, previous research (transforming the flow into dynamics and conversely) offers a partial explanation of some disasters such as catastrophic winds (tornadoes, typhoons), catastrophic floods, crashes without an apparent cause of aircraft etc. Indeed, the geometry of space created by nature or by human activities, conscious or not, generates gyroscopic forces that lead to spiraling (uncontrolled) evolutions. Some of the catastrophes can be avoided by using sensors that highlight spiral or exotic movements.

If in a flow we have chaos (sensitive dependence on initial conditions, three or more dimensions), there is chaos in the associated geometric dynamics. The Riemannian structure can generate also chaos in geometric dynamics.

Author Contributions: Conceptualization, C.U. and I.T.; methodology, C.U. and I.T.; writing-original draft preparation, C.U. and I.T.; writing-review and editing, C.U. and I.T. All authors have read and agreed to the published version of the manuscript.

Funding: This research received no external funding.

Acknowledgments: The authors are indebted to the Mathematics referees and to Valeriu Prepelita and Brandusa Prepelita-Raileanu who insisted on getting an improved version both scientifically and linguistically. Scientifically supported by University Politehnica of Bucharest and by Academy of Romanian Scientists.

Conflicts of Interest: The authors declare no conflict of interest.

\section{References}

1. Isvoranu, D.; Udriste, C. Fluid flow versus Geometric Dynamics. BSG Proceed. 2006, 13, 70-82. 
2. Udriste, C. Geometric Dynamics; Mathematics and Its Applications (Book 513); Kluwer Academic Publishers: Dordrecht, The Netherlands, 2000.

3. Udriste, C. Geometric Dynamics. SEA Bull. Math. 2000, 24, 313-322. [CrossRef]

4. Udriste, C.; Postolache, M. Atlas of Magnetic Geometric Dynamics; Monographs and Textbooks 3; Geometry Balkan Press: Bucharest, Romania, 2001.

5. Udriste, C.; Ferrara, M.; Opris, D. Economic Geometric Dynamics; Monographs and Textbooks 6; Geometry Balkan Press: Bucharest, Romania, 2004.

6. Udriste, C.; Ciancio, A. Linearized Geometric Dynamics of Tobin-Benhabib-Miyao economic flow. Balkan J. Geom. Appl. 2004, 9, 125-130.

7. Udriste, C. Geodesic motion in a gyroscopic field of forces. Tensor. New Ser. 2005, 66, 215-228.

8. Udriste, C.; Bejenaru, A. Invex energies on Riemannian manifolds. In Dynamical Systems and Methods; Luo, A.C.J., Machado, J.A.T., Balenu, D., Eds.; Springer-Verlag: New York, NY, USA, 2012; pp. 191-214.

9. Palis, J., Jr. Geometric Dynamics: Proceedings of the International Symposium, held at Instituto de Matematica Pura e Aplicada, Rio de Janeiro, Brasil, July-August 1981; Springer-Verlag: Berlin, Germany, 1983.

10. Gay-Balmaz, F.; Holm, D.D.; Ratiu, T.S. Geometric dynamics of optimization. Commun. Math. Sci. $2009,11$. [CrossRef]

11. Stefanescu, S.; Udriste, C. Magnetic field lines around filiform electrical circuits of right angle type. U.P.B. Sci. Bull. Ser. A 1993, 55, 3-18.

12. Treanta, S.; Udriste, C. On Auto-Parallel behavior of some special plane or space curves. In Proceedings of the 17th International Conference on Systems, Control, Signal Processing and Informatics (SCSI'13), Rhodes Island, Greece, 16-19 July 2013; pp. 90-94.

13. Furi, M. Second order differential equations on manifolds and forced oscillations. In Topological Methods in Differential Equations and Inclusions; Springer: Dordrecht, The Netherland, 1995.

14. Mihlin, S.G. Linear Partial Differential Equations; Encyclopedic and Scientific Editorial House: Bucharest, Romania, 1983. (In Romanian)

15. Udriste, C.; Teleman, A.M. Hamiltonian approaches of Field theory. IJMMS 2004, 57, 3045-3056. [CrossRef]

16. Arnold, V.I. Hamiltonian nature of Euler equations of rigid solid and ideal fluid dynamics. Uspehi Matem. Nauk. 1969, 24, 225-226.

17. Chorin, A.J.; Marsden, J.E. A Mathematical Introduction to Fluid Mechanics; Springer-Verlag: New York, NY, USA, 2000.

18. Udriste, C.; Udriste, A. From flows and metrics to dynamics and winds. Bull. Cal. Math. Soc. 2006, 98, 389-394.

19. Schubert, G.; Counselman, C.C.; Hansen, J.; Limaye, S.S.; Pettengill, G.; Seiff, A.; Shapiro, I.I.; Suomi, V.E.; Taylor, F.; Travis, L.; et al. Dynamics, winds, circulation and turbulence in the atmosphere of Venus. Space Sci. Rev. 1977, 20, 357-387. [CrossRef]

20. Lovelock, D.; Rund, H. Tensors, Differential Forms, and Variational Principles; Wiley: Hoboken, NJ, USA, 1975.

21. Samuelson, P.A. Law of conservation of the capital-output ratio. Proc. Nat. Acad. Sci. USA 1970, 67, 1477-1479. [CrossRef] [PubMed]

22. Samuelson, P.A. Law of conservation of the capital-output ratio in closed von Neumann systems. In Conservation Laws and Symmetry, Applications to Economics and Finance; Satao, R., Ramachandran, R.V., Eds.; Springer: Dordrecht, The Netherland, 1990; pp. 53-56.

(C) 2020 by the authors. Licensee MDPI, Basel, Switzerland. This article is an open access article distributed under the terms and conditions of the Creative Commons Attribution (CC BY) license (http://creativecommons.org/licenses/by/4.0/). 